\title{
ON THE DUAL VARIABLE OF THE CAUCHY STRESS TENSOR IN ISOTROPIC FINITE HYPERELASTICITY
}

\author{
CLAUDE VALLEE, DANIELLE FORTUNE, AND CAMELIA LERINTIU
}

\begin{abstract}
Elastic materials are governed by a constitutive law linking the second Piola-Kirchhoff stress tensor $\Sigma$ and the right Cauchy-Green strain tensor $C=F^{T} F$. Isotropic elastic materials are the special ones for which the Cauchy stress tensor $\sigma$ depends solely of the left Cauchy-Green strain tensor $B=F F^{T}$. In this paper we revisit the following property of isotropic hyperelastic materials: if the constitutive law linking $\Sigma$ and $C$ derives from a potential $\alpha$, then $\sigma$ and $\ln B$ are linked by a constitutive law deriving from the potential $\alpha \circ$ exp. We give a new and concise proof which is based on an explicit formula expressing the derivative of the exponential of a tensor.
\end{abstract}

\section{INTRODUCTION}

According to the mass conservation principle, the mass density per unit volume $\rho$ and its initial value $\rho_{0}$ are in the ratio

$$
\frac{\rho_{0}}{\rho}=\operatorname{det} F=(\operatorname{det} C)^{\frac{1}{2}}=(\operatorname{det} B)^{\frac{1}{2}} .
$$

The relation

$$
(\operatorname{det} F) \sigma=F \Sigma F^{T}
$$

between the Cauchy stress tensor $\sigma$ and the second Piola-Kirchhoff stress tensor $\Sigma$ can be rewritten

$$
\frac{\sigma}{\rho}=F \frac{\Sigma}{\rho_{0}} F^{T} .
$$

Let us agree to formulate the elastic materials constitutive laws as:

$$
\frac{\Sigma}{\rho_{0}}=h(C) \text {. }
$$

The polar decomposition $F=R U$ of the deformation gradient [3] implies:

$$
B=F F^{T}=R U^{2} R^{T}=R C R^{T} \text { or } C=R^{T} B R .
$$

This allows to translate the relation between $\Sigma$ and $C$ by a law satisfied by $\sigma$ :

$$
\frac{\sigma}{\rho}=R U h\left(R^{T} B R\right) U R^{T}=\left(R U R^{T}\right) R h\left(R^{T} B R\right) R^{T}\left(R U R^{T}\right)
$$

where we have enlightened the tensor $R U R^{T}$ which is nothing else than the square root $B^{\frac{1}{2}}$ of the positive definite symmetric tensor $B$. A priori, for elastics materials, the tensor $\frac{\sigma}{\rho}$ is a function of $B$ and $R$ :

$$
\frac{\sigma}{\rho}=B^{\frac{1}{2}} R h\left(R^{T} B R\right) R^{T} B^{\frac{1}{2}} .
$$

It will depend solely of $B$ in a single case: when the tensor $\operatorname{Rh}\left(R^{T} B R\right) R^{T}$ does not depend on the rotation $R$. The rotations forming a group, the only possible 
tensorial functions $h$ are those satisfying the relations of isotropy with respect to $B$ :

$$
R h\left(R^{T} B R\right) R^{T}=h(B) \quad \text { or } \quad R^{T} h(B) R=h\left(R^{T} B R\right) .
$$

Because of the relation $C=R^{T} B R$, the isotropy of the function $h$ can alternatively be expressed with respect to $C$ :

$$
R h(C) R^{T}=h\left(R C R^{T}\right) \quad \text { or } \quad R^{T} h\left(R C R^{T}\right) R=h(C) .
$$

To summarize: if the law $\frac{\Sigma}{\rho_{0}}=h(C)$ is isotropic, then $\frac{\sigma}{\rho}$ depends only of $B$, and it is the sole case; furthermore, under this isotropy condition

$$
\frac{\sigma}{\rho}=B^{\frac{1}{2}} h(B) B^{\frac{1}{2}}
$$

In this paper, we revisit the property of isotropic hyperelastic materials for which the existence of a potential expressing the constitutive law between $\frac{\Sigma}{\rho_{0}}$ and $C$ implies the existence of a potential linking $\frac{\sigma}{\rho}$ and $\ln B$.

\section{Isotropy of THE CONSTitutive LAW LINKING $\frac{\sigma}{\rho}$ AND $B$}

Let $\Omega$ be a rotation, if we change $B$ into $\Omega^{T} B \Omega$, then $B^{\frac{1}{2}}$ is changed into $\Omega^{T} B^{\frac{1}{2}} \Omega$ and $\frac{\sigma}{\rho}$ is changed in:

$$
\Omega^{T} B^{\frac{1}{2}} \Omega h\left(\Omega^{T} B \Omega\right) \Omega^{T} B^{\frac{1}{2}} \Omega=\Omega^{T} B^{\frac{1}{2}} h(B) B^{\frac{1}{2}} \Omega=\Omega^{T} \frac{\sigma}{\rho} \Omega .
$$

The isotropy of the constitutive law linking $\frac{\Sigma}{\rho_{0}}$ and $C$ is thus transferred to the constitutive law linking $\frac{\sigma}{\rho}$ and $B$.

\section{Coaxiality of $B$ and $h(B)$}

Theorem 3.1. Because $h$ is isotropic, the symmetric tensors $B$ and $h(B)$ are coaxial (i.e. they have the same eigenvectors).

Proof. Let $n$ be an eigenvector of $B$ chosen unitary, and let us consider the rotation of angle $\pi$ around $n$ :

$$
S=(\cos \pi) I+(1-\cos \pi) n n^{T}=2 n n^{T}-I
$$

with $I$ as the identity tensor. Such a symmetry $S$ leaves $n$ unchanged and changes any orthogonal vector to $n$ in its opposite. The tensor $B$ being symmetric, its other two eigenvectors are orthogonal to $n$, as a consequence $S^{T} B S=B$.

The isotropy condition implies $S^{T} h(B) S=h\left(S^{T} B S\right)$ or $h(B) S=S h(B)$, therefore $h(B)[S n]=S[h(B) n]$ or $S[h(B) n]=h(B) n$. Since the sole vectors unchanged by $S$ are the vectors parallel to $n$, the last equality is possible only when the vector $h(B) n$ remains parallel to the vector $n$, that is to say when $n$ is also an eigenvector for $h(B)$.

We easily deduce from this coaxiality property the two next corollaries, which will reveal important in the following.

Corollary 3.2. $B$ and $h(B)$ commute.

Corollary 3.3. For every real number $s, h(B)$ commutes with the power $B^{s}$ of $B$.

The choice $s=\frac{1}{2}$ allows one to simplify the expression $\frac{\sigma}{\rho}=B^{\frac{1}{2}} h(B) B^{\frac{1}{2}}$ in

$$
\frac{\sigma}{\rho}=h(B) B \text {. }
$$




\section{Hyperelastic materials}

4.1. Existence of a potential between the second Piola-Kirchhoff stress tensor $\Sigma$ and the right Cauchy-Green strain tensor $C$. Let us consider a derivable function $\alpha$ of $C$, its derivative $D \alpha(C)$ is a linear mapping from the space of symmetric tensors to $\mathbb{R}$. Thus, there exists a symmetric tensor denoted $\frac{\partial \alpha}{\partial C}$ such that for every variation $\delta C$ of $C$ :

$$
D \alpha(C) \delta C=\operatorname{tr}\left(\frac{\partial \alpha}{\partial C} \delta C\right) .
$$

Hyperelastic materials are those for which there exists a function $\alpha$ such that

$$
\frac{\Sigma}{\rho_{0}}=\frac{\partial \alpha}{\partial C}
$$

In this assumption, we will say that the constitutive law linking the tensors $\frac{\Sigma}{\rho_{0}}$ and $C$ derives from the potential $\alpha$.

4.2. Derivative of the exponential of a matrix. Let us consider a square ma$\operatorname{trix} A$ and a real number $t$, the exponential $\exp (t A)$ is the solution of the matricial ordinary differential equation

$$
\frac{d}{d t} \exp (t A)=A \exp (t A)
$$

which is equal to $I$ at $t=0$. Let $\delta A$ be a variation of $A$, in the varied equation

$$
\frac{d}{d t} D(\exp )(t A)(t \delta A)=\delta A[\exp (t A)]+A D(\exp )(t A)(t \delta A)
$$

let us introduce the square matrix $M(t)$ defined by

$$
D(\exp )(t A)(t \delta A)=[\exp (t A)] M(t)
$$

The varied equation becomes

$$
\left[\frac{d}{d t} \exp (t A)\right] M(t)+[\exp (t A)] \frac{d M}{d t}=\delta A[\exp (t A)]+A[\exp (t A)] M(t)
$$

and simplifies itself into the ordinary differential equation

$$
\frac{d M}{d t}=[\exp (-t A)] \delta A[\exp (t A)]
$$

which can be integrated by quadrature. Because $M(0)$ vanishes, we easily deduce from it the value of $M(1)$ and thereafter the variation of the exponential of a matrix [10]:

$$
D(\exp )(A)(\delta A)=[\exp (A)] \int_{0}^{1}[\exp (-s A)] \delta A[\exp (s A)] d s .
$$

In the special case where $A$ is the logarithm of the positive definite tensor $B$, this formula allows us to predict that for every variation $\delta B$ of $B$ :

$$
D(\exp )(\ln B) \delta B=B \int_{0}^{1} B^{-s} \delta B B^{s} d s
$$




\subsection{Existence of a potential between the Cauchy stress tensor and the logarithm of the left Cauchy-Green strain tensor.}

Theorem 4.1. If the tensor $\frac{\Sigma}{\rho_{0}}$ derives from a potential $\alpha$ of the tensor $C$, then the tensor $\frac{\sigma}{\rho}$ derives from the potential $\alpha$ oxp of the tensor $\ln B$.

Proof. By deriving the compound function $\alpha \circ \exp$, we find successively:

$$
\begin{gathered}
D(\alpha \circ \exp )(\ln B) \delta B=D \alpha(B)(D(\exp )(\ln B) \delta B) \\
=\operatorname{tr}\left(\frac{\partial \alpha}{\partial B}[D(\exp )(\ln B) \delta B]\right)=\operatorname{tr}\left(h(B) B \int_{0}^{1} B^{-s} \delta B B^{s} d s\right)=\int_{0}^{1} \operatorname{tr}\left[h(B) B B^{-s} \delta B B^{s}\right] d s .
\end{gathered}
$$

To simplify the last integral, it is necessary to pay attention on the switchings because the matrix $\delta B$ does not commute with the others. However, under the trace, we can make cross at the beginning the last term of the product of 5 matrices. Then from Corollary 2, we can switch this term $B^{s}$ with $h(B)$ and afterwards with $B$, it ends up just before $B^{-s}$. The product of the two matrices $B^{s}$ and $B^{-s}$ reduces to the identity tensor $I$, and the integral simplifies itself into

$$
\operatorname{tr}(h(B) B \delta B)=\operatorname{tr}\left(\frac{\sigma}{\rho} \delta B\right) .
$$

The final value of the integral allows to conclude to the constitutive law:

$$
\frac{\sigma}{\rho}=\frac{\partial(\alpha \circ \exp )}{\partial(\ln B)}
$$

\section{Conclusion}

Without resorting to the Taylor expansion of the logarithm [11] or of the exponential [8] of a symmetric tensor, nor to its spectral decomposition [5], we have given an intrinsic proof of the existence of the potential $\alpha \circ \exp$ between $\frac{\sigma}{\rho}$ and $\ln B$. Numerous isotropic hyperelastic constitutive laws expressing directly $\sigma$ in term of $\ln B$ have been proposed ([2], [6], [7, [9], 12]) and numerically implemented [4. When the potential $\alpha \circ \exp$ is convex, the consideration of its Legendre-FenchelMoreau transform is a tool to perform the inversion of the constitutive law (1), 13, [14), ie to express the Hencky logarithmic strain tensor $\ln B$ in term of the Cauchy stress tensor $\sigma$.

\section{REFERENCES}

[1] Blume, J.A.: On the form of the inverted stress-strain law for isotropic hyperelastic solids. International Journal of Non-Linear Mechanics. 27(3), 413-421 (1992)

[2] Bruhns, O.T., Xiao, H., Meyers, A.: Constitutive inequalities for an isotropic elastic strainenergy function based on Hencky's logarithmic strain tensor. Proceedings of the Royal Society of London, Series A-Mathematical Physical and Engineering Sciences. 457(2013), 2207-2226 (2001)

[3] Ciarlet, P.G.: Mathematical Elasticity, vol.1. 3D Elasticity, North-Holland, Amsterdam (1988)

[4] Feng, Z.-Q., Vallée, C., Fortuné, D., Peyraut, F.: The 3e hyperelastic model applied to the modeling of 3D impact problems. Finite Elements in Analysis and Design. 43(1), 51-58 (2006)

[5] Hoger, A.: The stress conjugate to logarithmic strain. International Journal of Solids and Structures. 23(12), 1645-1656 (1987)

[6] Ogden, R.W., Saccomandi, G., Sgura, I.: Fitting hyperelastic models to experimental data. Computational Mechanics. 34(6), 484-502 (2004) 
[7] Peric, D., Owen, D.R.J., Honnor, M.E.: A model for Finite Strain elastoplasticity based on logarithmic strains - computational issues. Computer Methods in Applied Mechanics and Engineering. 94(1), 35-61 (1992)

[8] Sansour C.: On the dual variable of the logarithmic strain tensor, the dual variable of the Cauchy stress tensor, and related issues. International Journal of Solids and Structures. 38(50-51), 9221-9232 (2001)

[9] Sendova, T., Walton, J.R.: On strong ellipticity for isotropic hyperelastic materials based upon logarithmic strain. International Journal of Non-Linear Mechanics. 40(2-3), 195-212 (2005)

[10] Souriau, J.M.: Calcul linéaire. P.U.F, Paris (1959)

[11] Vallée, C.: Laws of isotropic hyperelastic behaviour (in french). International Journal of Engineering Science. 16(7), 451-457 (1978)

[12] Xiao, H., Chen, L.S.: Hencky's elasticity model and linear stress-strain relations in isotropic finite hyperelasticity. Acta Mechanica. 157(1-4), 51-60 (2002)

[13] Xiao, H., Chen, L.S.: Hencky's logarithmic strain and dual stress-strain and strain-stress relations in isotropic finite hyperelasticity. International Journal of Solids and Structures. 40(6), 1455-1463 (2003)

[14] Xiao, H., Bruhns, O.T., Meyers, A: Explicit dual stress-strain and strain-stress relations of incompressible isotropic hyperelastic solids via deviatoric Hencky strain and Cauchy stress. Acta Mechanica. 168(1-2), 21-33 (2004)

Laboratoire de Mécanique des Solides, UMR CNRS 6610, Université de Poitiers, SP2Mi, Téléport 2, Boulevard Marie et Pierre Curie, B.P. 30179, 86962, FuturoscopeChasseneuil Cedex, France, Tel.: 0033-(0)549-496798, Fax: 0033-(0)549-496791

E-mail address: vallee@lms.univ-poitiers.fr

Laboratoire de Mécanique des Solides, UMR CNRS 6610, Université de Poitiers, SP2MI, Téléport 2, Boulevard Marie et Pierre Curie, B.P. 30179, 86962, FuturoscopeChasseneuil Cedex, France

Laboratoire de Mécanique des Solides, UMR CNRS 6610, Université de Poitiers, SP2Mi, Téléport 2, Boulevard Marie et Pierre Curie, B.P. 30179, 86962, FuturoscopeChasseneuil Cedex, France 